\title{
A Virtual Cardiovascular Care Program for Prevention of Heart Failure Readmissions in a Skilled Nursing Facility Population: Retrospective Analysis
}

Daniel M Friedman ${ }^{1,2^{*}}$, BA; Jana M Goldberg ${ }^{1 *}$, MD; Rebecca L Molinsky ${ }^{3}$, MPH; Mark A Hanson ${ }^{1,4}$, PhD; Adam Castaño $^{1}$, MD, MS; Syed-Samar Raza ${ }^{5}$, MD; Nodar Janas ${ }^{1,5}$, MD; Peter Celano ${ }^{1}$, MBA; Karen Kapoor ${ }^{1}$, BS; Jina Telaraja $^{1}$, MS, PAC; Maria L Torres ${ }^{1}$, BS; Nayan Jain ${ }^{1}$, BS; Jeffrey D Wessler ${ }^{1,6}$, MD, MPhil

${ }^{1}$ Heartbeat Health, Inc., New York, NY, United States

${ }^{2}$ Vagelos College of Physicians \& Surgeons, Columbia University, New York, NY, United States

${ }^{3}$ Division of Epidemiology \& Community Health, School of Public Health, University of Minnesota, Minneapolis, MN, United States

${ }^{4}$ Innovative Practice \& Telemedicine Section, Department of Emergency Medicine, The George Washington University, Washington, DC, United States

${ }^{5}$ Cassena Care, LLC, Woodbury, NY, United States

${ }^{6}$ Division of Cardiology, Department of Medicine, Columbia University Irving Medical Center, New York, NY, United States

*these authors contributed equally

\section{Corresponding Author:}

Daniel M Friedman, BA

Heartbeat Health, Inc.

156 W 56th Street

Suite 1000

New York, NY, 10019

United States

Phone: 18668265888

Email: daniel@heartbeathealth.com

\begin{abstract}
Background: Patients with heart failure (HF) in skilled nursing facilities (SNFs) have 30-day hospital readmission rates as high as $43 \%$. A virtual cardiovascular care program, consisting of patient selection, initial televisit, postconsultation care planning, and follow-up televisits, was developed and delivered by Heartbeat Health, Inc., a cardiovascular digital health company, to 11 SNFs (3510 beds) in New York. The impact of this program on the expected SNF 30-day HF readmission rate is unknown, particularly in the COVID-19 era.
\end{abstract}

Objective: The aim of the study was to assess whether a virtual cardiovascular care program could reduce the 30-day hospital readmission rate for patients with HF discharged to SNF relative to the expected rate for this population.

Methods: We performed a retrospective case review of SNF patients who received a virtual cardiology consultation between August 2020 and February 2021. Virtual cardiologists conducted 1 or more telemedicine visit via smartphone, tablet, or laptop for cardiac patients identified by a SNF care team. Postconsult care plans were communicated to SNF clinical staff. Patients included in this analysis had a preceding index admission for HF.

Results: We observed lower hospital readmission among patients who received 1 or more virtual consultations compared with the expected readmission rate for both cardiac (3\% vs $10 \%$, respectively) and all-cause etiologies (18\% vs $27 \%$, respectively) in a population of 3510 patients admitted to SNF. A total of $185 / 3510$ patients $(5.27 \%)$ received virtual cardiovascular care via the Heartbeat Health program, and 40 patients met study inclusion criteria and were analyzed, with 26 (65\%) requiring 1 televisit and $14(35 \%)$ requiring more than 1 . Cost savings associated with this reduction in readmissions are estimated to be as high as US $\$ 860$ per patient.

Conclusions: The investigation provides initial evidence for the potential effectiveness and efficiency of virtual and digitally enabled virtual cardiovascular care on 30-day hospital readmissions. Further research is warranted to optimize the use of novel virtual care programs to transform delivery of cardiovascular care to high-risk populations. 


\section{KEYWORDS}

heart failure; readmissions; skilled nursing facilities; posthospitalization; cardiovascular; cardiology; outcome; cost; virtual care; telehealth; telemedicine; mobile health; mHealth; digital health

\section{Introduction}

\section{Background}

Heart failure (HF) is the leading cause of hospitalization and readmission in the Medicare population [1]. Among the more than 1.5 million residents within skilled nursing facilities (SNFs) in the United States, 20\%-37\% carry a diagnosis of HF [2]. In the current era, 1 in 4 older patients hospitalized with HF is discharged to a SNF [3]. SNFs operate on transfer agreement(s) with 1 or more participating hospitals to provide skilled nursing, medical care, and rehabilitation services for patients that are injured, disabled, or sick [4].

HF readmission rates, while high at baseline, are even higher within the SNF population. Although community HF readmission rates average $22 \%, 30$-day $\mathrm{HF}$ readmission rates in the SNF setting range from $27 \%$ to $43 \%[3,5,6]$. There is great interest in reducing this "revolving door" phenomenon within the growing SNF population, as patients are living longer with greater disease severity and multiple comorbidities [4,7]. These readmissions also come at great expense to the health care system, averaging over US $\$ 9000$ for a typical HF readmission [8].

Virtual visits have been identified as a potential solution to provide access to health care populations at high risk for readmission, such as those with HF. A pilot study conducted by the Cleveland Clinic examined the feasibility and safety of substituting in-person visits with virtual visits for patients with HF transitioning from hospital to home [9]. The authors found that there were no significant differences in hospital readmissions, emergency room visits, or death between the 2 groups. The no-show rate with virtual visits also trended lower than the rate for in-person visits [10].

\section{The Impact and Resilience of Virtual Cardiovascular Care}

The COVID-19 pandemic propelled virtual care to center stage in 2020 given the need to reduce exposure risk among both health care workers and patients, particularly in the SNF setting. For the first time ever, virtual visits surpassed in-person visits in percentage of overall visit volume. Survey data reported that virtual visits ballooned from $9 \%$ of patient interaction prepandemic to $51 \%$ at its peak [11]. Although televisits declined in subsequent months, virtual care has had significant staying power and will likely outlast the pandemic, as supported by recent Centers for Medicare and Medicaid Services (CMS) telehealth expansion [12]. In the 2021 Physician Fee Schedule, CMS has expanded telehealth coverage, reducing frequency limitations on telehealth services in nursing facilities, allowing for more frequent virtual visits to occur should they be indicated [13]. Further, the Federal Communications Commission has announced additional funding to explore Connected Care Pilot Programs [14].

There are often barriers to access to specialty cardiology care when a patient is in the SNF setting, which may be reduced by virtual care [9]. Virtual visits may eliminate overhead required for an in-person visit from a SNF setting, such as preparing the patient for transfer via ambulance or ambulette and receiving them upon their return. In-person clinic visits may expose already high-risk patients to infectious diseases, including SARS-CoV-2 and typical seasonal illnesses such as influenza. Given the susceptibility of SNF settings to outbreaks of contagious illnesses, the elimination of unnecessary exposure has the potential to benefit both residents and staff. Such visits may not only reduce readmissions, but also reduce costly emergency department visits that do not ultimately result in admission. Potential benefits to virtual cardiology care in the SNF setting are summarized in Table 1.

Despite concerns about physical examination limitations during virtual care, developing data suggest that remote assessment of jugular venous pressure may correlate with invasive right heart catheterization measurements. In one study, bedside and remote jugular venous pressure assessments were comparable and significantly correlated with invasive measurements [15]. Other modified maneuvers can be performed during a virtual examination, such as an assessment for lower extremity edema. To observe any edema, the provider may ask a patient by video to show their legs and press on them, any indentation suggesting pitting edema. If patients are in a SNF at the time of virtual visit, staff nurses may assist patients with their examination, helping them to overcome any barriers due to mobility. Further, the ability for a staff member to assist a patient with a virtual examination is invaluable given the existing disparities in the telehealth space, particularly in the Medicare or Medicaid population. These patients may have limited access to computers with an internet connection or smartphones [16]. 
Table 1. Benefits of virtual cardiology care in the SNF ${ }^{\mathrm{a}}$ setting [9].

\begin{tabular}{|c|c|}
\hline Group & Potential benefits \\
\hline - SNF patients & $\begin{array}{ll}\text { - } & \text { Increased access to timely care } \\
\text { - } & \text { Increased access to follow-up visits } \\
\text { - } & \text { Reduced disruption (ie, no transport) } \\
\text { - } & \text { Reduced exposure to infectious diseases (eg, flu, SARS-CoV-2) } \\
\text { - } & \text { Reduced time to optimize guideline-directed medical theory }\end{array}$ \\
\hline - $\quad$ Providers & $\begin{array}{ll}\text { - } & \text { Increase care delivery to high-risk population } \\
\text { - } & \text { Reduced disruption to clinic flow } \\
\text { - } & \text { Increased feasibility of frequent follow-up televisits } \\
\text { - } & \text { History can be supplemented by SNF nurse during televisit } \\
\text { - } & \text { Physical examination can be performed by a SNF nurse during televisit }\end{array}$ \\
\hline - $\quad$ Skilled nursing facility & $\begin{array}{ll}\text { - } & \text { Reduced disruption (ie, no transport) } \\
\text { - } & \text { Reduced exposure to infectious diseases (eg, flu, SARS-CoV-2) for all residents } \\
\text { - } & \text { Reduced reimbursable days lost to readmission }\end{array}$ \\
\hline - $\quad$ Payers & - Reduced costs (readmission, transportation) \\
\hline - Health care system & $\begin{array}{ll}\text { - } & \text { Reduced costs } \\
\text { - } & \text { Support research efforts }\end{array}$ \\
\hline
\end{tabular}

${ }^{\mathrm{a}} \mathrm{SNF}$ : skilled nursing facility.

\section{Purpose}

Recent paradigm shifts in policies and attitudes toward virtual care have opened the door for new methods of care delivery, particularly in high-risk populations. While both specialty and virtual care delivery have been points of interest for posthospitalization populations, access challenges, including technology literacy and familiarity, have prevented widespread adoption of such approaches. Introduction of virtual cardiovascular care into the SNF setting offers a compelling opportunity to address these challenges. Given the increase in complex, older patients living with HF transitioning from hospitals to SNFs, this population may stand to benefit from novel, digitally enabled care pathways.

The purpose of this study was to assess the feasibility, efficacy, and efficiency of virtual cardiovascular care delivery on a population of patients with a preceding index admission for $\mathrm{HF}$ in a SNF setting. We hypothesized that a virtual cardiovascular care program involving at least one virtual consultation would reduce the 30-day readmission rate for patients with $\mathrm{HF}$ relative to the expected rate for this population for reasons including the following: (1) direct communication of a care plan between the patient and specialist, (2) specialist inference of gaps in the patient's medical history from the visit and chart review, and (3) titration of pharmacotherapies. Relatedly, the introduction of virtual care within the controlled and assisted environment of a SNF could help to bridge the technology literacy and familiarity divide that is associated with the study demographic, in addition to saving costs attributed to prevented hospital readmissions. This investigation precedes an expected larger study of payer-referred postacute patients along with a future randomized control trial targeting similar high cardiovascular risk patients.

\section{Methods}

\section{Study Overview}

This study represents an uncontrolled initial exploration conducted over 7 months across a population of patients receiving long-term care and subacute rehabilitation in 11 SNFs and 3510 beds in the New York metropolitan area. A total of 185 patients were evaluated based on their referral by the SNF staff. The SNF care team identified patients with a diagnosis of congestive HF and initiated program enrollment by scheduling a virtual consultation (consult) with a cardiologist on the Heartbeat Health software platform. Following enrollment and participation in a virtual cardiovascular care program, retrospective chart reviews were conducted across all study participants and results were tabulated with a specific focus on hospital readmissions and related outcome improvements and estimated cost savings.

\section{Virtual Cardiovascular Care Technology Platform}

Virtual cardiovascular care was enabled by a software technology platform provided by Heartbeat Health, a digital health company with a focus on virtual cardiovascular care delivery. The platform consists of a software provider interface and patient facilitator interface. The interfaces are linked by a secure cloud-based infrastructure connecting the experiences. Both of the experiences, provider and patient, can be delivered over mobile, tablet, or laptop device viewports. The technology allows for patient enrollment, patient or patient-assisted requests for care, provider review of patient records, instantaneous virtual video and voice visits, and ongoing patient-provider coordination, among other capabilities. The platform can be used in conjunction with electronic medical record of the SNF or practice. The technology was deployed in a provider setting with a group of cardiologists overseeing care and in a SNF 
setting with the clinical staff, including nurses and nurse managers, assisting SNF patients in the delivery of care.

\section{Virtual Cardiovascular Care Program}

Virtual cardiovascular care was made available to patients with a variety of cardiac conditions, though only patients with HF were included in this study. An initial online request for virtual cardiovascular care was submitted to Heartbeat Health by a representative from the SNF. Pertinent cardiac symptoms and diagnoses were noted in the request alongside any additional notable context. The first consult was scheduled within 1-2 business days of this request. Consults were performed by a remote cardiologist with a SNF nurse at the patient's bedside. Postconsult care plans were provided to the SNF clinical staff for implementation. Some patients required additional consults that were scheduled by the cardiologist as clinically indicated. Consults were typically focused on symptom assessment, volume management, and maximization of guideline-directed medical therapy (GDMT). Heartbeat Health and the cardiology consult team were not involved in the decision to send patients to the hospital for readmission; SNF clinical teams made this decision autonomously on an as-needed basis (Figure 1).

Figure 1. A flowchart of the virtual cardiovascular care program for patients. HBH: Heartbeat Health; SNF: skilled nursing facility.

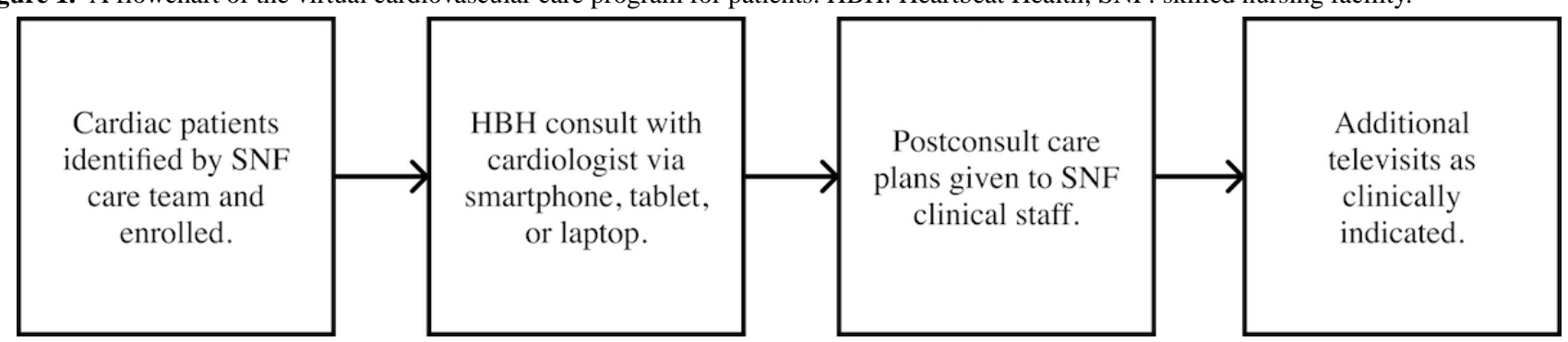

We performed a retrospective case review of cardiovascular consultations that occurred between August 2020 and February 2021. Data were deidentified and aggregated for analysis. Inclusion criteria included an index hospitalization for $\mathrm{HF}$, either $\mathrm{HF}$ with reduced ejection fraction (HFrEF) or HF with preserved ejection fraction (HFpEF), an initial virtual consultation within 30 days of arrival to the SNF, and not receiving a comfort care protocol. Patients who were discharged home from the SNF setting within 30 days of arrival were considered lost to follow-up, as patient readmission status was determined using SNF data after 30 days from the date of admission.

In a population of 3510 SNF beds, 185 patients received virtual cardiology care via the Heartbeat Health program. A total of 45 patients met inclusion criteria, of which 40 were analyzed and 5 were lost to follow-up, as they were discharged from SNF to home within 30 days of their hospitalization (Figure 2).

Figure 2. Patient flow through the analysis. CHF: congestive heart failure, SNF: skilled nursing facility.

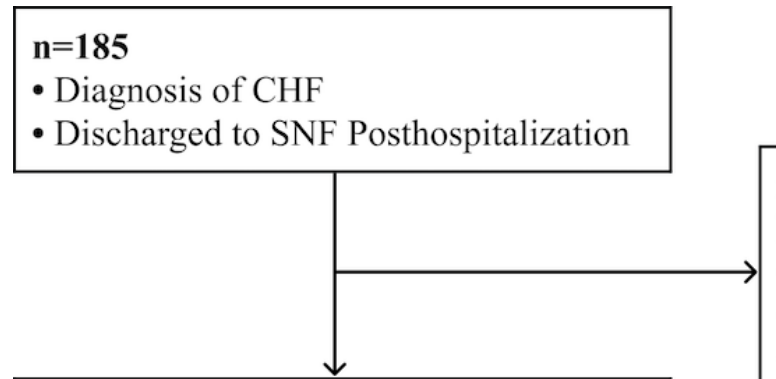

$n=45$

- Enrolled in virtual cardiology program

\section{$\mathrm{n}=\mathbf{1 4 0}$}

Excluded

- Index admission not due to CHF

- Consultation occurred more than 30 days after SNF arrival

- Receiving comfort care protocol

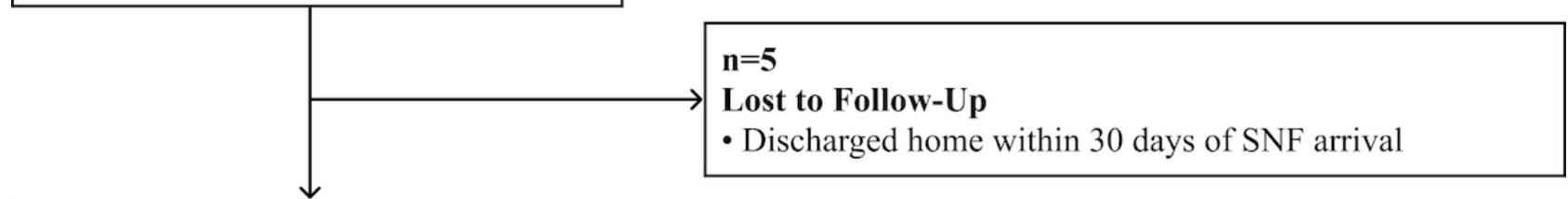

$\mathrm{n}=\mathbf{4 0}$

- Analyzed

\section{Statistical Analysis}

All analyses were conducted using $\mathrm{R}$ (version 4.0.3; $\mathrm{R}$ Foundation for Statistical Analysis). Participant baseline characteristics are presented as percentages or means (SD). A retrospective analysis was conducted comparing readmission status among participants. Percentages or means (SE) were computed across readmission status. $P$ values for difference among readmission status were obtained from Fisher exact test or analysis of variance tests. Hedges $g$ (a corrected version of Cohen $d$ for smaller sample sizes) and 95\% CIs were computed to assess effect size for continuous risk factors. Lastly, odds ratios and $95 \%$ CIs across readmission status are presented for categorical risk factors. 


\section{Results}

At baseline, patients were on average 80.5 (SD 10.4) years old, $50 \%$ (20/40) were female, 65\% (26/40) White, 28\% (11/40) Black, 5\% (2/40) Hispanic, and 3\% (1/40) Asian (Table 2). In accordance with recent literature, race should be viewed as a proxy metric for social, environmental, and structural factors rather than as a biological risk factor [17]. Baseline comorbidities were typical of an older SNF population with HF and included coronary artery disease $(17 / 40,43 \%)$, hypertension $(38 / 40,95 \%)$, diabetes $(10 / 40,25 \%)$, and a history of stroke $(4 / 40,10 \%)$. Mean ejection fraction was $41.6 \%$ (SD 17.9), with approximately $40 \%(16 / 40)$ of patients having HFpEF, 53\% (21/40) having HFrEF, and the remaining 8\% (3/40) having unspecified HF. Patients in New York Heart Association (NYHA) Class II or Class III were classified together and represented $93 \%(37 / 40)$ of patients. No patients were NYHA Class I, and 8\% (3/40) were NYHA Class IV. 
Table 2. Baseline characteristics.

\begin{tabular}{|c|c|}
\hline Characteristic & Values $(\mathrm{N}=40)$ \\
\hline Age (years), mean (SD) & $80.5(10.4)$ \\
\hline Ejection fraction (\%), mean (SD) & $41.6(17.9)$ \\
\hline Systolic blood pressure $(\mathrm{mmHg})$, mean $(\mathrm{SD})$ & $127.5(19.4)$ \\
\hline Diastolic blood pressure (mmHg), mean (SD) & $67.7(9.6)$ \\
\hline \multicolumn{2}{|l|}{$\operatorname{Sex}, \mathbf{n}(\%)$} \\
\hline Male & $20(50)$ \\
\hline Female & $20(50)$ \\
\hline \multicolumn{2}{|l|}{ Race, $\mathrm{n}(\%)$} \\
\hline Black & $11(28)$ \\
\hline Hispanic & $2(5)$ \\
\hline Asian & $1(3)$ \\
\hline White & $26(65)$ \\
\hline \multicolumn{2}{|l|}{$\mathrm{HF}^{\mathrm{a}}$ type, $\mathrm{n}(\%)$} \\
\hline HF with reduced ejection fraction & $21(53)$ \\
\hline HF with preserved ejection fraction & $16(40)$ \\
\hline Unknown & $3(8)$ \\
\hline Coronary artery disease, $\mathrm{n}(\%)$ & $17(43)$ \\
\hline Stroke, n (\%) & $4(10)$ \\
\hline Hypertension, n (\%) & $38(95)$ \\
\hline Diabetes, n (\%) & $10(25)$ \\
\hline Chronic kidney disease, n (\%) & $15(38)$ \\
\hline Serum creatinine, mean (SD) & $1.64(1)$ \\
\hline Chronic obstructive pulmonary disease, n (\%) & $5(13)$ \\
\hline \multicolumn{2}{|l|}{ New York Heart Association Class, n (\%) } \\
\hline I & $0(0)$ \\
\hline II-III & $37(93)$ \\
\hline IV & $3(8)$ \\
\hline $\mathrm{ACEI}^{\mathrm{b}}, \mathrm{ARB}^{\mathrm{c}}$, or $\mathrm{ARNI}^{\mathrm{d}}, \mathrm{n}(\%)$ & $11(28)$ \\
\hline Beta blocker, n (\%) & $28(70)$ \\
\hline Loop diuretic, n (\%) & $36(90)$ \\
\hline Aldosterone inhibitor, n (\%) & $7(18)$ \\
\hline Intravenous inotrope, $\mathrm{n}(\%)$ & $3(8)$ \\
\hline
\end{tabular}

${ }^{\mathrm{a}} \mathrm{HF}$ : heart failure.

bACEI: angiotensin-converting enzyme inhibitor.

${ }^{\mathrm{c}} \mathrm{ARB}$ : angiotensin receptor blocker.

${ }^{\mathrm{d}}$ ARNI: angiotensin receptor-neprilysin inhibitor.

Of the 40 patients analyzed, 1 patient (3\%) was readmitted for a cardiac cause, as compared with the usual care readmission rate of $10 \%$ for this population (Figure 3) [18]. Seven patients (18\%) had all-cause readmissions (inclusive of the 1 cardiac readmission), as compared with the usual care readmission rate of $27 \%$ for this population [3].

A total of $26 / 40$ patients $(65 \%)$ required only 1 virtual consultation, whereas $14 / 40(35 \%)$ required more than 1 consultation as requested by SNF medical staff or cardiology discretion (Figure 4). One patient required 7 consults during the 30-day period. Additional consults were most commonly called for volume management followed by blood pressure control. 
Retrospective associations between readmission status and patient characteristics are shown in Table 3. Significant differences were found between those readmitted and those not readmitted with respect to age $(P=.04)$, race $(P=.04)$, and number of consults $(P=.006)$. Mean age (SE) among those readmitted was significantly lower compared with those who were not readmitted (73 [4.3] vs 82 [1.7], $P=.04$ ). Readmission status also significantly differed by race $(P=.04)$. Specifically, when comparing readmission status among Black and White patients,

Figure 3. Readmission rate by cause $[3,15]$. the odds of readmission among the former was 9.21 times the odds of readmission among the latter $(P=.01,95 \% \mathrm{CI}$ 1.17-119.50). Lastly, the mean number of consults among those readmitted was significantly higher compared with those who were not readmitted $(P=.01)$.

Of the patients readmitted, the majority were diagnosed with $\operatorname{HFrEF}(5 / 7,71 \%)$ and had at least two consults $(5 / 7,71 \%)$, though each patient had a unique reason for readmission (Table 4).

\section{Virtual Care Usual Care}

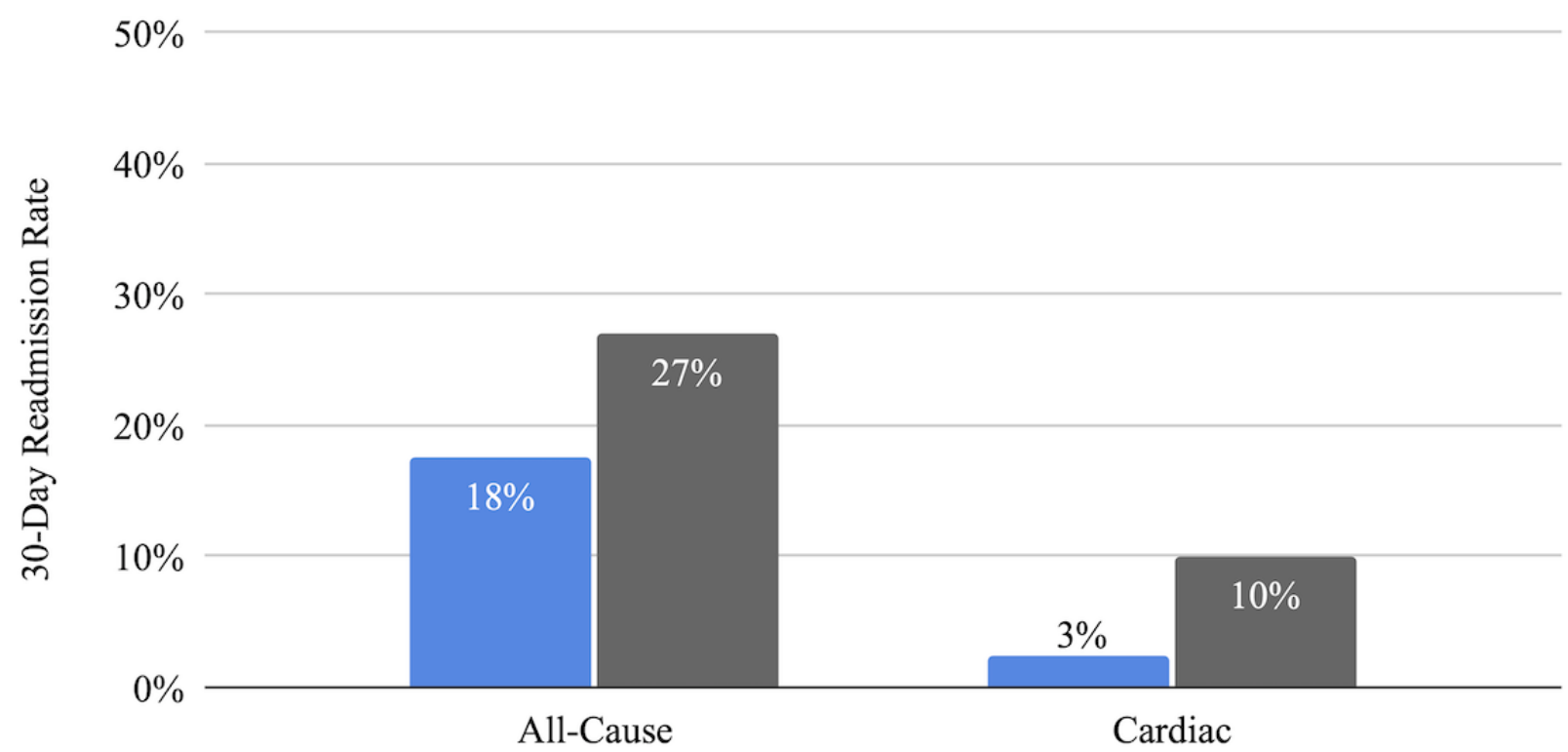

Readmission Category 
Figure 4. Number of virtual consults per patient.

30

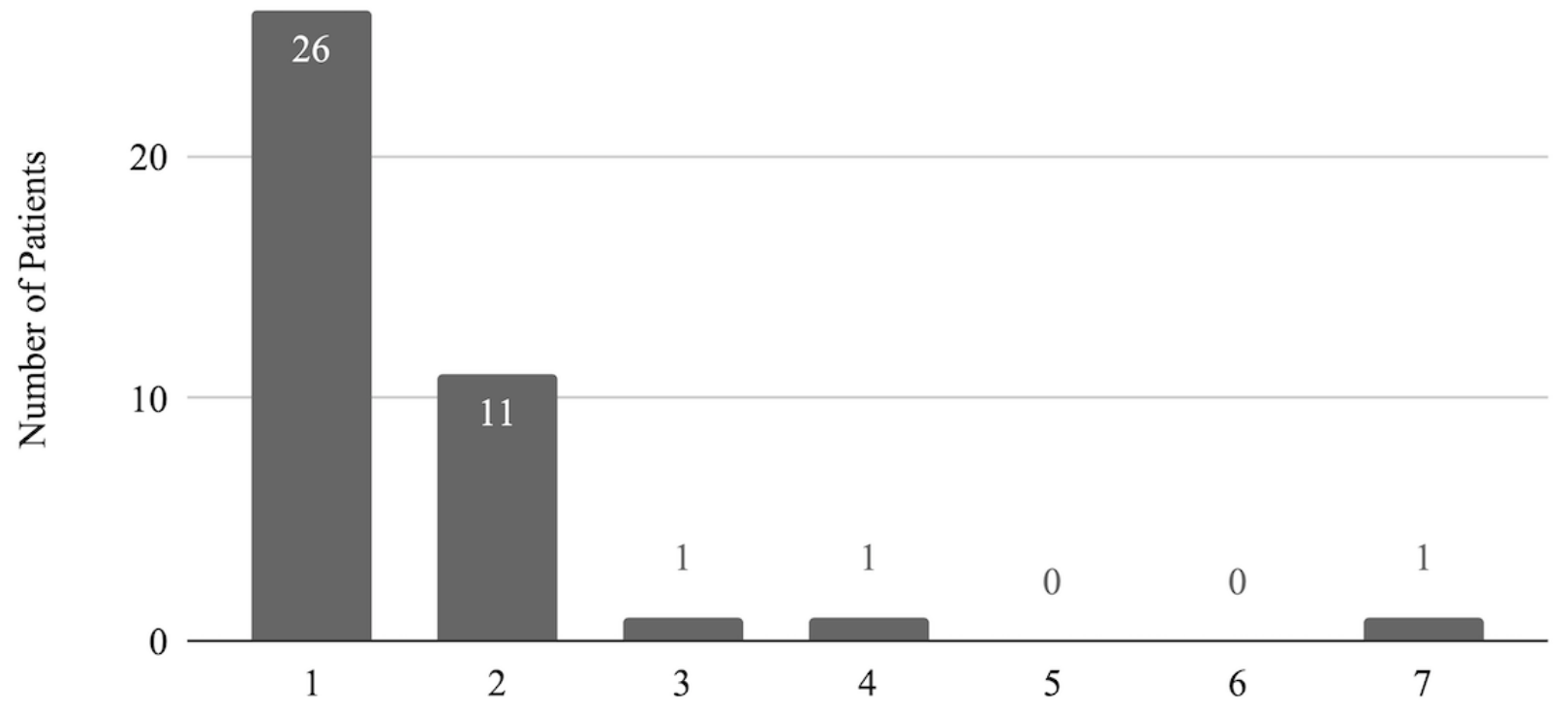

Consults Per Patient 
Table 3. Retrospective associations between hospital readmission and characteristics of participants $(n=40)$.

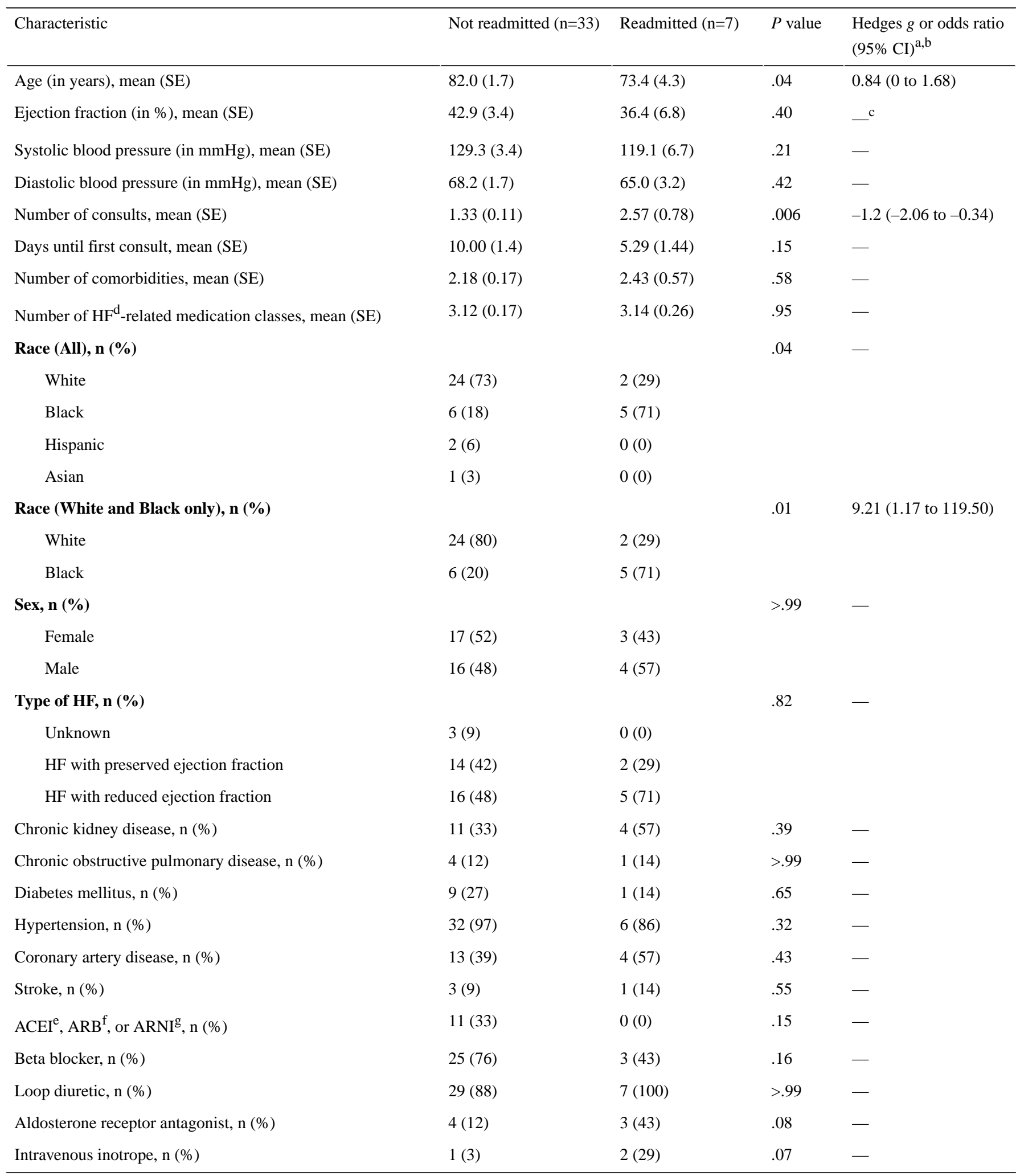

${ }^{\mathrm{a} U n p a i r e d} t$ tests and Hedges $g$ test were performed for continuous outcomes along with $95 \% \mathrm{CI}$.

${ }^{\mathrm{b}}$ Fisher exact test and odds ratios were performed for categorical variables along with $95 \% \mathrm{CI}$.

c__: Not available

${ }^{\mathrm{d}} \mathrm{HF}$ : heart failure.

eACEI: angiotensin-converting enzyme inhibitor.

${ }^{\mathrm{f}} \mathrm{ARB}$ : angiotensin receptor blocker.

g ARNI: angiotensin receptor-neprilysin inhibitor. 
Table 4. Characteristics of patients readmitted within 30 days.

\begin{tabular}{|c|c|c|c|}
\hline Readmission category & Readmission reason & Days in skilled nursing facility before readmission, $\mathrm{n}$ & Total consults, $n$ \\
\hline Cardiac & Volume overload & 15 & 2 \\
\hline Noncardiac & Pneumonia (unspecified) & 11 & 1 \\
\hline Noncardiac & Pneumonia (COVID-19) & 7 & 2 \\
\hline Noncardiac & Fever, hypoxia & 22 & 2 \\
\hline Noncardiac & Pleural effusion & 30 & 3 \\
\hline Noncardiac & Acute kidney injury & 20 & 7 \\
\hline Noncardiac & Mechanical fall & 26 & 1 \\
\hline
\end{tabular}

\section{Discussion}

\section{Principal Findings}

The results of this study suggest that patients with HF who are discharged to SNFs and receive at least one virtual cardiology consultation within 30 days may have lower cardiac-related and all-cause 30-day readmission rates than the expected rates for this population.

There may be several reasons for the reduction in cardiac readmissions as evidenced by clinical literature exploring cardiovascular care in SNF settings. There may be a lack of familiarity among SNF clinical teams with current $\mathrm{HF}$ guidelines, which are complex and regularly evolving [5,19]. HF care plans are synthesized from data surrounding most recent left ventricular function assessment, weight trends, and parameters for uptitration of GDMT. However, measurement of weight may be a particular barrier as many SNFs have a standard protocol to weigh patients weekly, which is discordant with the daily weight trending required for many patients with HF [20]. Further, regular SNF diets may be high in sodium (>2000 mg per day), which can add to sodium retention if an order for a low-sodium diet is not clear [5]. SNFs may have limited cardiac records accessible to them upon transfer. While admission documentation may typically include physical and social information pertinent to rehabilitation, HF details and guidance on management may be scant or absent [5].

Specialty cardiologist supervision, delivered virtually, can provide a backstop of care to support HF protocols in SNF settings. The most obvious benefit of such supervision relates to the increase in access of patients to a care specialty at the time of need. Often, cardiovascular care in the SNF setting is delayed beyond advisable timeframes, resulting in less desirable outcomes and even heightened readmission risk. This guidance, critical for a successful transition of care, can be provided through a virtual cardiology consultation when otherwise unavailable from transfer documents. Access to a cardiologist who is well-versed with the necessity for uptitration of GDMT, as well as volume management, is irreplaceable in high-risk patients. Although most patients in this study only required 1 virtual consultation, repeat visits, often needed for GDMT titration, were made significantly more feasible given their virtual nature. The frequent touchpoints may have been a contributing factor to the reduced readmission rate observed in this analysis.
There were some notable differences in characteristics of patients that were readmitted to the hospital compared with those who were not. There were significantly more consultations performed on patients who were readmitted, which may be indicative of the level of acuity among these patients. While the additional visits may result in a slightly higher cost of care for these patients, the approach is compatible with GDMT and holds further outcome and cost benefits to HF population management through a reduction in readmissions. There were no significant differences in readmissions between patients with HFrEF and those with HFpEF, which is consistent with prior studies that have found similar rates of readmission between both HFrEF and HFpEF [21]. Comorbidities were also similar between those readmitted and those who were not. In terms of medical therapy, patients on intravenous inotropes trended toward readmission, though it did not reach statistical significance.

Notably, there was a significant difference between White and Black patients, with Black patients significantly more likely to be readmitted (odds ratio 9.21, 95\% CI 1.17-119.50). This disparity has been identified in previous research examining HF readmissions in a large municipal health system, with Black patients having a higher odds ratio for 90-day readmissions than White patients (odds ratio 1.21, 95\% CI 1.01-1.47) [22]. Given the known racial disparities in health care, particularly those facing the Black community, more research should be performed to identify the role for virtual care in actively closing existing care gaps and combating institutional racism [17].

\section{Limitations}

This study presents an initial investigation into the relationship between virtual cardiovascular care delivery in SNF settings and overall readmissions, and subsequent work will address several known limitations to these findings. First, we compared our readmission rates with the expected readmission rates for the greater SNF population rather than baseline readmission data from the specific nursing facilities evaluated, which would have been preferred as a comparison. Second, patients who were discharged from the SNF within their 30-day readmission window were lost to follow-up. Because we were unable to capture readmission data from this group, we excluded them from analysis, introducing selection bias. Although a discharge to a private home is likely a favorable prognostic indicator, the 30-day readmission status of these patients who underwent the virtual cardiology program remains unknown. Third, the strength of this study was limited by its small sample size. In the future, 
we hope to replicate this study as a randomized control trial with a larger population of SNF patients, which will address several limitations including selection bias, confounding, generalizability, and consistency.

\section{Efficiency Gains of Virtual Cardiovascular Care}

There are significant implications to consider with regard to cost and time when one replaces an ambulatory in-person visit from a SNF with a virtual one. Based on current CMS transitional care guidelines, an in-person visit is required within the first 7-14 calendar days of discharge (CPT 99495) [23]. Traditional in-person appointments come at great expense to the long-term care facility and, in turn, the health care system. In-person visits come with direct costs (eg, ambulance- or ambulette-based transportation service, accompanying transport staff) and indirect costs (eg, time spent by nurses to prepare a patient for transfer). The average scheduled visit in this study was 15 minutes in length with no necessary transportation cost. In the traditional setting, the ambulance trip from an in-network transport firm averages about US $\$ 400$ (thus US $\$ 800$ roundtrip), and the visit itself represents some US \$200 in cost, for a total of US $\$ 1000$. This is 5 times more than when an in-person visit is substituted virtually [24,25]. Readmission is the most expensive avoidable outcome, with an average cost of US \$9051 per HF readmission [8]. The decreased all-cause readmission rate of $17.5 \%$ for the virtual care group in this study represents an average expected cost reduction of US $\$ 860$ per patient (Table 5).

Table 5. Readmission rates of patients with heart failure from skilled nursing facility with virtual cardiology program versus usual care [3,8,18].

\begin{tabular}{|c|c|c|c|c|}
\hline \multirow[t]{2}{*}{ Readmissions impacts } & \multicolumn{2}{|c|}{ All cause (30 days) } & \multicolumn{2}{|c|}{ Cardiac (30 days) } \\
\hline & Usual care & Virtual care & Usual care & Virtual care \\
\hline Skilled nursing facility heart failure readmission rate, $\%$ & 27 & 17.5 & 10 & 2.5 \\
\hline Expected readmission cost per patient (US \$) & 2444 & 1584 & 905 & 226 \\
\hline Expected readmission savings per patient (US \$) & $\mathrm{N} / \mathrm{A}^{\mathrm{b}}$ & 860 & N/A & 679 \\
\hline
\end{tabular}

${ }^{a}$ Data surrounding average congestive heart failure readmission costs specifically due to cardiac etiologies were unavailable and thus assumed to be comparable with those of all-cause etiologies.

${ }^{\mathrm{b}}$ N/A: Not applicable.

\section{The Opportunities for Virtual Cardiovascular Care}

Virtual cardiovascular care is still in a nascent state and opportunities for its extension are numerous. The program could be expanded to include additional visits as needed from a generalist, with more of a focus on reducing all-cause readmissions. Further, more rigid protocols could be established for repeat virtual consults, with patients clinically stratified based on their readmission risk; higher-risk stratifications would warrant more frequent virtual consults as supported by GDMT protocols.

Future programs could enforce an initial cardiology consult within 7 days of SNF arrival. In this study, initial consults occurred on average 9 days after SNF arrival. Early postdischarge follow-up for patients is strongly associated with lower 30-day readmission. For instance, observational data examining administrative claims from hospitals of fee-for-service Medicare beneficiaries found that hospitals which had the lowest percentage of 1-week postdischarge follow-up rates after $\mathrm{HF}$ admissions had the highest 30-day readmission rates [26]. Another study investigated the postdischarge follow-up characteristics associated with 30-day readmission in patients with HF [27]. Researchers found that $50 \%$ of patients had clinical contact within 7 days $(84 \%$ of the contacts were done via in-person clinic visit versus $16 \%$ telephone calls). Patients who were followed up within 7 days postdischarge had a $19 \%$ lower adjusted odds ratio of readmission.

While televisits appear beneficial in the SNF setting, virtual care resources may also bring valuable structure to the home setting upon discharge, particularly in patients with HF. Weintraub et al [28] demonstrated that pairing remote patient monitoring (measuring heart rate, blood pressure, and weight) with an HF disease management program resulted in lower HF hospitalizations when compared with standard care.

\section{Conclusions}

The implementation of a virtual cardiovascular care program represents a promising way to reduce readmission rates in patients with HF in the SNF setting. Our findings and the discussion above should serve as a call to action for more research efforts examining postdischarge HF workflows within the virtual care space, particularly to challenge in-person requirements for transitional care management. Further research is warranted to determine how virtual care programs may not only provide additive benefit to existing care modalities, but also transform how care is delivered to improve outcomes, cost efficiency, and the overall care experience.

\section{Acknowledgments}

This work was supported by Heartbeat Health, Inc. and Cassena Care, LLC. RM was supported by institutional training grant (No. T32HL007779) from the National Institutes of Health. 


\section{Conflicts of Interest}

JW, MH, and NJai are founders of and receive salary or compensation from Heartbeat Health, Inc. DF, JG, AC, PC, KK, JT, NJan, and MT receive salary or compensation from Heartbeat Health, Inc. S-SR and NJan receive salary or compensation from Cassena Care, LLC. RM has no conflicts of interest to declare.

\section{References}

1. Jencks SF, Williams MV, Coleman EA. Rehospitalizations among patients in the Medicare fee-for-service program. N Engl J Med 2009 Apr 2;360(14):1418-1428. [doi: 10.1056/NEJMsa0803563] [Medline: 19339721]

2. Jurgens CY, Goodlin S, Dolansky M, Ahmed A, Fonarow GC, Boxer R, American Heart Association Council on Quality of CareOutcomes Researchthe Heart Failure Society of America. Heart failure management in skilled nursing facilities: a scientific statement from the American Heart Association and the Heart Failure Society of America. Circ Heart Fail 2015 May;8(3):655-687. [doi: 10.1161/HHF.0000000000000005] [Medline: 25855686]

3. Allen LA, Hernandez AF, Peterson ED, Curtis LH, Dai D, Masoudi FA, et al. Discharge to a skilled nursing facility and subsequent clinical outcomes among older patients hospitalized for heart failure. Circ Heart Fail 2011 May;4(3):293-300 [FREE Full text] [doi: 10.1161/CIRCHEARTFAILURE.110.959171] [Medline: 21447803]

4. Medicare Skilled Nursing Facility Manual. 2000. URL: https://www.cms.gov/Regulations-and-Guidance/Guidance/ Transmittals/downloads/R367SNF.pdf [accessed 2020-11-12]

5. Boxer RS, Dolansky MA, Frantz MA, Prosser R, Hitch JA, Piña IL. The Bridge Project: improving heart failure care in skilled nursing facilities. J Am Med Dir Assoc 2012 Jan;13(1):83.e1-83.e7 [FREE Full text] [doi:

10.1016/j.jamda.2011.01.005] [Medline: 21450244]

6. Bueno H, Ross JS, Wang Y, Chen J, Vidán MT, Normand ST, et al. Trends in length of stay and short-term outcomes among Medicare patients hospitalized for heart failure, 1993-2006. JAMA 2010 Jun 02;303(21):2141-2147 [FREE Full text] [doi: 10.1001/jama.2010.748] [Medline: 20516414]

7. Manemann SM, Chamberlain AM, Boyd CM, Weston SA, Killian J, Leibson CL, et al. Skilled Nursing Facility Use and Hospitalizations in Heart Failure: A Community Linkage Study. Mayo Clin Proc 2017 Mar 13:e1 [FREE Full text] [doi: 10.1016/j.mayocp.2017.01.014] [Medline: 28365097]

8. Mayr FB, Talisa VB, Balakumar V, Chang CH, Fine M, Yende S. Proportion and Cost of Unplanned 30-Day Readmissions After Sepsis Compared With Other Medical Conditions. JAMA 2017 Feb 07;317(5):530-531. [doi: 10.1001/jama.2016.20468] [Medline: 28114505]

9. Gorodeski EZ, Goyal P, Cox ZL, Thibodeau JT, Reay RE, Rasmusson K, et al. Virtual Visits for Care of Patients with Heart Failure in the Era of COVID-19: A Statement from the Heart Failure Society of America. J Card Fail 2020 Jul;26(6):448-456 [FREE Full text] [doi: 10.1016/j.cardfail.2020.04.008] [Medline: 32315732]

10. Gorodeski EZ, Moennich LA, Riaz H, Jehi L, Young JB, Tang WHW. Virtual Versus In-Person Visits and Appointment No-Show Rates in Heart Failure Care Transitions. Circ Heart Fail 2020 Aug;13(8):e007119. [doi:

10.1161/CIRCHEARTFAILURE.120.007119] [Medline: 32762457]

11. HHS Issues New Report Highlighting Dramatic Trends in Medicare Beneficiary Telehealth Utilization amid COVID-19. 2019 Jul 28. URL: https://www.hhs.gov/about/news/2020/07/28/

hhs-issues-new-report-highlighting-dramatic-trends-in-medicare-beneficiary-telehealth-utilization-amid-covid-19.html [accessed 2021-05-17]

12. Verma S. Early impact of CMS expansion of Medicare telehealth during COVID-19. Health Affairs. 2020 Jul 15. URL: https://www.healthaffairs.org/do/10.1377/hblog20200715.454789/full/ [accessed 2021-05-17]

13. Proposed Policy, Payment, and Quality Provisions Changes to the Medicare Physician Fee Schedule for Calendar Year 2021. 2020 Aug 3. URL: https://www.cms.gov/newsroom/fact-sheets/proposed-policy-payment-and-quality-provisionschanges-medicare-physician-fee-schedule-calendar-year-4 [accessed 2021-05-17]

14. Federal Communications Commission. Wireline Competition Bureau Announces Connected Care Pilot Program Application Filing Window Opening. 2020. URL: https://docs.fcc.gov/public/attachments/DA-20-1315A1.pdf [accessed 2021-05-17]

15. Kelly SA, Schesing KB, Thibodeau JT, Ayers CR, Drazner MH. Feasibility of Remote Video Assessment of Jugular Venous Pressure and Implications for Telehealth. JAMA Cardiol 2020 Jul 01:1194-1195. [doi: 10.1001/jamacardio.2020.2339] [Medline: $\underline{32609293]}$

16. Julien HM, Eberly LA, Adusumalli S. Telemedicine and the Forgotten America. Circulation 2020 Jul 28;142(4):312-314 [FREE Full text] [doi: 10.1161/CIRCULATIONAHA.120.048535] [Medline: 32525712]

17. Boyd R, Lindo E, Weeks L, McLemore M. On racism: a new standard for publishing on racial health inequities. Health Affairs Blog. $2020 \mathrm{Jul}$ 2. URL: https://www.healthaffairs.org/do/10.1377/hblog20200630.939347/full/ [accessed 2021-05-17]

18. Ogunneye O, Rothberg MB, Friderici J, Slawsky MT, Gadiraju VT, Stefan MS. The association between skilled nursing facility care quality and 30-day readmission rates after hospitalization for heart failure. Am J Med Qual 2015 May;30(3):205-213. [doi: 10.1177/1062860614531069] [Medline: 24736837] 
19. Orr NM, Boxer RS, Dolansky MA, Allen LA, Forman DE. Skilled Nursing Facility Care for Patients With Heart Failure: Can We Make It "Heart Failure Ready?". J Card Fail 2016 Dec;22(12):1004-1014 [FREE Full text] [doi: 10.1016/j.cardfail.2016.10.009] [Medline: 27769909]

20. Chaudhry SI, Wang Y, Concato J, Gill TM, Krumholz HM. Patterns of weight change preceding hospitalization for heart failure. Circulation 2007 Oct 2;116(14):1549-1554 [FREE Full text] [doi: 10.1161/CIRCULATIONAHA.107.690768] [Medline: 17846286]

21. Nichols GA, Reynolds K, Kimes TM, Rosales AG, Chan WW. Comparison of Risk of Re-hospitalization, All-Cause Mortality, and Medical Care Resource Utilization in Patients With Heart Failure and Preserved Versus Reduced Ejection Fraction. Am J Cardiol 2015 Oct 01;116(7):1088-1092. [doi: 10.1016/j.amjcard.2015.07.018] [Medline: 26235928]

22. Durstenfeld MS, Ogedegbe O, Katz SD, Park H, Blecker S. Racial and Ethnic Differences in Heart Failure Readmissions and Mortality in a Large Municipal Healthcare System. JACC Heart Fail 2016 Nov;4(11):885-893 [FREE Full text] [doi: 10.1016/j.jchf.2016.05.008] [Medline: 27395346]

23. January 2020 ASC Approved HCPCS code and Payment Rates. 2020. URL: https://www.cms.gov/Medicare/ Medicare-Fee-for-Service-Payment/HospitalOutpatientPPS/Annual-Policy-Files-Items/2020-Annual-Policy-Files [accessed 2020-11-18]

24. Ambulance Fee Schedule. 2020. URL: https://www.cms.gov/Medicare/Medicare-Fee-for-Service-Payment/ AmbulanceFeeSchedule/afspuf [accessed 2021-05-17]

25. US Government Accountability Office. Ambulance Providers: Costs and Medicare Margins Varied Widely; Transports of Beneficiaries Have Increased. 2012. URL: https://www.gao.gov/products/GAO-13-6 [accessed 2021-05-17]

26. Hernandez AF, Greiner MA, Fonarow GC, Hammill BG, Heidenreich PA, Yancy CW, et al. Relationship between early physician follow-up and 30-day readmission among Medicare beneficiaries hospitalized for heart failure. JAMA 2010 May 05;303(17):1716-1722. [doi: 10.1001/jama.2010.533] [Medline: 20442387]

27. Lee KK, Yang J, Hernandez AF, Steimle AE, Go AS. Post-discharge Follow-up Characteristics Associated With 30-Day Readmission After Heart Failure Hospitalization. Med Care 2016 Apr;54(4):365-372 [FREE Full text] [doi: 10.1097/MLR.0000000000000492] [Medline: 26978568]

28. Weintraub A, Gregory D, Patel AR, Levine D, Venesy D, Perry K, et al. A multicenter randomized controlled evaluation of automated home monitoring and telephonic disease management in patients recently hospitalized for congestive heart failure: the SPAN-CHF II trial. J Card Fail 2010 Apr;16(4):285-292. [doi: 10.1016/j.cardfail.2009.12.012] [Medline: 20350694]

\section{Abbreviations}

ACEI: angiotensin-converting enzyme inhibitor

ARB: angiotensin receptor blocker

ARNI: angiotensin receptor-neprilysin inhibitor

GDMT: guideline-directed medical therapy

HF: heart failure

HFpEF: heart failure with preserved ejection fraction

HFrEF: heart failure with reduced ejection fraction

NYHA: New York Heart Association

SNF: skilled nursing facility

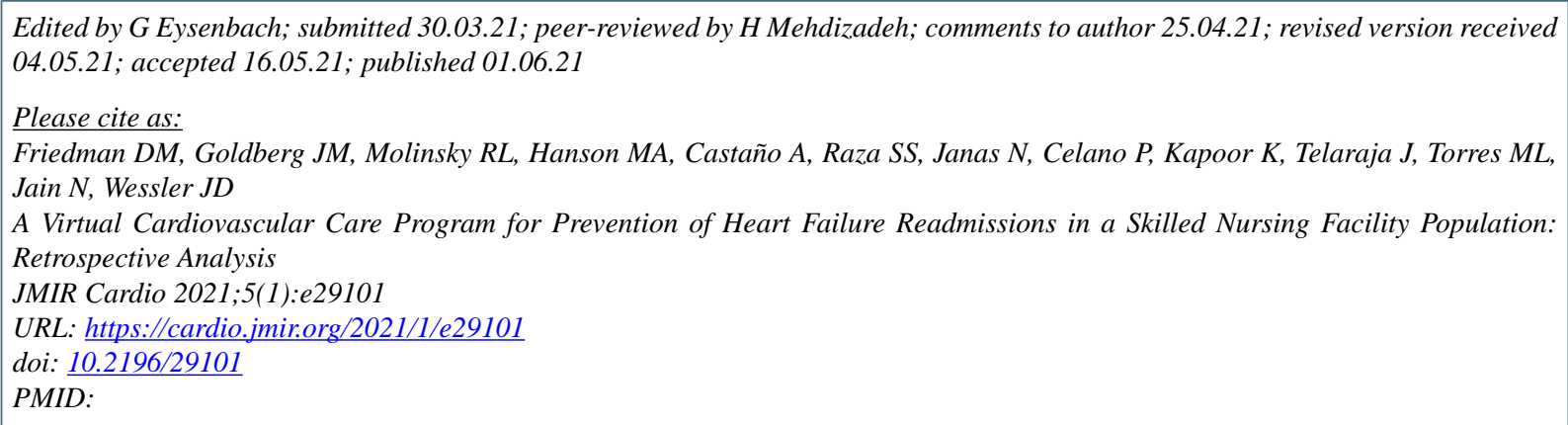

CDaniel M Friedman, Jana M Goldberg, Rebecca L Molinsky, Mark A Hanson, Adam Castaño, Syed-Samar Raza, Nodar Janas, Peter Celano, Karen Kapoor, Jina Telaraja, Maria L Torres, Nayan Jain, Jeffrey D Wessler. Originally published in JMIR Cardio 
(https://cardio.jmir.org), 01.06.2021. This is an open-access article distributed under the terms of the Creative Commons Attribution License (https://creativecommons.org/licenses/by/4.0/), which permits unrestricted use, distribution, and reproduction in any medium, provided the original work, first published in JMIR Cardio, is properly cited. The complete bibliographic information, a link to the original publication on https://cardio.jmir.org, as well as this copyright and license information must be included. 